\title{
STAR-INVERTIBILITY AND $t$-FINITE CHARACTER IN INTEGRAL DOMAINS
}

\author{
CARMELO ANTONIO FINOCCHIARO, GIAMPAOLO PICOZZA, \\ AND FRANCESCA TARTARONE
}

\begin{abstract}
Let $A$ be an integral domain. We study new conditions on families of integral ideals of $A$ in order to get that $A$ is of $t$-finite character (i.e., each nonzero element of $A$ is contained in finitely many $t$-maximal ideals). We also investigate problems connected with the local invertibility of ideals.
\end{abstract}

\section{INTRODUCTION}

In their series of papers about flatness of ideals $([7,8])$, S. Glaz and $\mathrm{W}$. Vasconcelos address the problem of when flat ideals are invertible. Among other results, they conjecture that $([7, \S 3])$ :

(C1) "In an H-domain (i.e. a domain in which t-maximal ideals are divisorial), faithfully flat ideals are projective".

Since, for ideals in a domain, projective is equivalent to invertible and, by a result of D.D. Anderson and D.F. Anderson [2], faithfully flat is equivalent to locally principal, the condition "faithfully flat ideals are projective" can be restated as "locally principal ideals are invertible". This last condition appears again in a paper by S. Bazzoni [4], who conjectures that:

(C2) "Prüfer domains with this property have the finite character on maximal ideals".

In [13], the conjectures $(\mathrm{C} 1)$ and $(\mathrm{C} 2)$ are put in relation and it is shown that they are incompatible. Moreover, an example in which (C1) fails is exhibited. In the same time, several proofs of Bazzoni's conjecture appeared: W.C. Holland, J. Martinez, W. Wm. Mc Govern and M. Tesemma provided a proof of the original conjecture ([10]), relying strongly on methods of the theory of lattice ordered groups (their proof has been recently translated in a ring theoretic language

Date: June 4, 2018.

2000 Mathematics Subject Classification. Primary: 13A15, 13F05; Secondary: 13B30, $13 \mathrm{G} 05$.

Key words and phrases Finite character, invertible ideal, star-operation. 
in [12]); F. Halter-Koch gave a proof in the more general context of $r$ Prüfer monoids [9]; M. Zafrullah [15] also studied Bazzoni's conjecture for P $v$ MD's but developing different techniques from F. Halter Koch's paper.

In this paper, we further generalize these results. As pointed out in [13], the finite character on maximal ideal is not necessary to have that locally principal ideals are invertible. In fact, Noetherian domains have obviously this property, but they have not necessarily the finite character. However, Noetherian domains have the $t$-finite character, which turns out to be a sufficient condition to obtain the invertibility of locally principal ideals (on the other hand, Example 2.3 shows that $t$-finite character is not necessary, answering a question posed in [13]).

So, as in F. Halter-Koch and M. Zafrullah's papers ([15, 9]), we focus on $t$-versions of Bazzoni's conjecture. We study the following three different conditions, which are all equivalent to the condition "each locally principle ideal is invertible" in the case of Prüfer domains:

1) $t$-locally $t$-finite ideals are $t$-finite;

2) $t$-locally principal ideals are $t$-invertible;

3) $t$-locally $t$-invertible $t$-ideals are $t$-invertible;

All conditions 1)-3) hold when the domain has the $t$-finite character. Conversely we prove that condition 1) implies the $t$-finite character with the fairly mild hypothesis of the $t$-local $v$-coherence of the domain (Theorem 1.11).

Finally, with similar techniques, we show that condition 2) implies the $t$-finite character in domains which are locally GCD (Proposition 2.6) and that condition 3) implies the $t$-finite character in domains which are locally $\mathrm{P} v \mathrm{MD}$ (Proposition 2.8).

Briefly, we recall some terminology and definitions that we will freely use in the paper.

Let $A$ be an integral domain with quotient field $K$. Recall that a star operation on $A$ is a map $\star$ from the set $F(A)$ of nonzero fractional ideals of $A$ in itself, $I \mapsto I^{\star}$, verifying the following properties for all $I, J \in F(A), 0 \neq x \in K$ :

(a) $A^{\star}=A,(x I)^{\star}=x I^{\star}$;

(b) $I \subseteq J \Rightarrow I^{\star} \subseteq J^{\star}$;

(c) $\left(I^{\star}\right)^{\star}=I^{\star}$.

A star operation $\star$ on $A$ is said finite type star operation if for all $I \in F(A)$,

$$
I^{\star}=\bigcup\left\{J^{\star}: J \subseteq I, J \text { is finitely generated }\right\} .
$$


An ideal $I \in F(A)$ such that $I^{\star}=I$ is called a $\star$-ideal. As usual, $I^{-1}:=(A: I)=\{x \in K ; x I \subseteq A\}$, and $I$ is $\star$-invertible if $\left(I I^{-1}\right)^{\star}=$ $A$. An ideal $I$ is $\star$-finite if there exists a finitely generated ideal $J$ such that $J^{\star}=I^{\star}$. If $\star$ is of finite type, $J$ can always be taken inside $I$. A $\star$-invertible ideal is $\star$-finite.

If $\star$ is a finite type star operation, the set of $\star$-ideals has (proper) maximal elements, which are prime ideals and are called $\star-$ maximal ideals (this set is denoted by $\star-\operatorname{Max}(A)$ ). Moreover, every integral $\star$-ideal is contained in a $\star$-maximal ideal. We say that $A$ has the $\star$-finite character if each $\star$-ideal (equivalently, each element) of $A$ is contained in finitely many $\star-$ maximal ideals.

We will call an integral ideal of $A$ simply ideal. We will also denote by $\mathcal{I}^{\star}(A)$ (resp. $\mathcal{I}_{\text {fin }}^{\star}(A)$ ) the collection of all $\star$-ideals (resp. $\star$-finite $\star$-ideals) of $A$.

The identity is a finite type star operation and it is usually denoted by $d$. Other classical examples of star operations are:

- the $v$-operation, defined by $I \mapsto I^{v}=(A:(A: I))=\left(I^{-1}\right)^{-1}$;

- the $t$-operation, defined by

$$
I \mapsto I^{t}=\bigcup\left\{J^{v}: J \subseteq I, J \text { is finitely generated }\right\} .
$$

The $t$-operation is of finite type and it is maximal in a way that if $\star$ is another finite type star operation on $A$, then $I^{\star} \subseteq I^{t}$ for each $I \in F(A)$.

\section{Comaximal Families of iDEAls}

If $\mathscr{F}$ is a nonempty collection of subsets of a set, we denote by $\bigcap \mathscr{F}$ the intersection of all the members of $\mathscr{F}$. Moreover, if $\mathscr{F}$ is a finite collection of ideals of a ring, we denote by $\prod \mathscr{F}$ the product of all members of $\mathscr{F}$.

If $(X, \leq)$ is a partially ordered set, we shall denote by $\operatorname{Max}_{\leq}(X)$ the set of all the maximal elements of $X$. With these notation, if $\star$ is of finite type, then we have that $\left.\star-\operatorname{Max}(A)=\operatorname{Max}_{\subseteq}\left(\mathcal{I}^{\star}(A) \backslash\{A\}\right)\right)$.

If $E$ is a subset of $A, V(E)$ denotes the set of all the prime ideals of $A$ containing $E$.

Definition 1.1. Let $A$ be an integral domain and $\star$ a star operation on $A$.

(i) We say that an ideal $\mathfrak{a}$ of $A$ satisfies the property ( $\star$-c.a.) ( closure under addition) if, for each pair $\mathfrak{a}_{1}, \mathfrak{a}_{2}$ of proper $\star$-finite $\star$-ideals of $A$ containing $\mathfrak{a}$, then $\left(\mathfrak{a}_{1}+\mathfrak{a}_{2}\right)^{\star}$ is still a proper ideal of $A$. 
(ii) If $\mathscr{F}$ is a collection of ideals of $A$ and $a \in A \backslash\{0\}$, we say that $\mathscr{F}$ is a a $\star$-comaximal collection over $a$ if $a \in \bigcap \mathscr{F}$ and $\left(\mathfrak{a}_{1}+\mathfrak{a}_{2}\right)^{\star}=A$, for every $\mathfrak{a}_{1}, \mathfrak{a}_{2} \in \mathscr{F}$, with $\mathfrak{a}_{1} \neq \mathfrak{a}_{2}$.

Lemma 1.2. Let $A$ be an integral domain and $\star$ be a finite type star operation on $A$. Assume that $\mathfrak{a}$ is a proper $\star$-finite $\star$-ideal satisfying the property ( $\star-c . a$.$) . Then$

$$
\mathfrak{m}_{\mathfrak{a}}:=\left\{x \in A:((x)+\mathfrak{a})^{\star} \subsetneq A\right\}
$$

is the only $\star-$ maximal ideal containing $\mathfrak{a}$.

Proof. It is clear that $\mathfrak{m}_{\mathfrak{a}}$ is the union of the set of the $\star$-maximal ideals containing $\mathfrak{a}$. So, let $x, y \in \mathfrak{m}_{\mathfrak{a}}$. Since $\mathfrak{a}$ satisfies the $\star$-c.a. property, $(((x)+\mathfrak{a})+((y)+\mathfrak{a}))^{\star}$ is a proper $\star$-ideal, so it is contained in a $\star$-maximal ideal $\mathfrak{m} \in V(\mathfrak{a})$. Thus, $x, y \in \mathfrak{m}$ and so $x-y \in \mathfrak{m} \subseteq \mathfrak{m}_{\mathfrak{a}}$. That $a x \in \mathfrak{m}_{\mathfrak{a}}$, for all $a \in A, x \in \mathfrak{m}_{\mathfrak{a}}$ is straightforward. So $\mathfrak{m}_{\mathfrak{a}}$ is an ideal of $A$. Moreover, if $\mathfrak{b}=\left(b_{1}, b_{2}, \ldots, b_{n}\right)$ is a finitely generated ideal contained in $\mathfrak{m}_{\mathfrak{a}}$, we have that for each $i=1,2, \ldots, n, b_{i}$ is contained in a $\star$-maximal ideal $\mathfrak{m}_{i} \in V(\mathfrak{a})$. So $\left(\left(b_{i}\right)+\mathfrak{a}\right)^{\star} \subsetneq A$. Since $\mathfrak{a}$ satisfies the property $\star$-c.a., it follows that $(\mathfrak{b}+\mathfrak{a})^{\star} \subsetneq A$. Thus, $\mathfrak{b} \subseteq \mathfrak{m}$ for some $\mathfrak{m} \in V(\mathfrak{a})$. So we have that $\mathfrak{b}^{\star} \subseteq \mathfrak{m}^{\star}=\mathfrak{m} \subseteq \mathfrak{m}_{\mathfrak{a}}$, and so $\mathfrak{m}_{\mathfrak{a}}$ is a $\star$-ideal ( since $\star$ is of finite type).

So, it follows that $\mathfrak{m}_{\mathfrak{a}}$ is a $\star$-ideal containing all the $\star$-maximal ideals that contain $\mathfrak{a}$, that is $\mathfrak{m}_{\mathfrak{a}}$ is a $\star$-maximal ideal and it is the only one that contains $\mathfrak{a}$.

Let $A$ be an integral domain and $a$ be a nonzero element of $A$. We denote by $\left(\Sigma^{\prime}\right)_{a}^{\star}$ the collection of all the $\star$-comaximal families over $a$ of proper $\star$-finite $\star$-ideals of $A$. We call $\Sigma_{a}^{\star}$ the collection of all the $\star$-comaximal families over $a$ of proper $\star$-finite $\star$-ideals of $A$ satisfying the property ( $\star$-c.a.).

Obviously, we have the containment $\Sigma_{a}^{\star} \subseteq\left(\Sigma^{\prime}\right)_{a}^{\star}$.

Proposition 1.3. Let $A$ be an integral domain, $\star$ a finite type star operation on $A$ and $a \in A \backslash\{0\}$. Assume that there exists a finite collection of ideals $\mathscr{F} \in \Sigma_{a}^{\star} \cap \operatorname{Max}_{\subseteq}\left(\left(\Sigma^{\prime}\right)_{a}^{\star}\right)$. Then $V(a) \cap \star-\operatorname{Max}(A)=$ $\left\{\mathfrak{m}_{\mathfrak{a}}: \mathfrak{a} \in \mathscr{F}\right\}\left(\mathfrak{m}_{\mathfrak{a}}\right.$ as in Lemma 1.2), and, in particular, it is finite.

Proof. Let $\mathscr{F}:=\left\{\mathfrak{a}_{1}, \ldots, \mathfrak{a}_{n}\right\}$. By Lemma 1.2, the inclusion $V(a) \cap$ $\star-\operatorname{Max}(A) \supseteq\left\{\mathfrak{m}_{\mathfrak{a}_{1}}, \ldots, \mathfrak{m}_{\mathfrak{a}_{n}}\right\}$ is immediate. Conversely, assume, by contradiction, that there exists a $\star$-maximal ideal $\mathfrak{m}$ containing a such that $\mathfrak{m} \neq \mathfrak{m}_{\mathfrak{a}_{1}}, \ldots, \mathfrak{m}_{\mathfrak{a}_{n}}$. In particular, we have $\mathfrak{m} \nsubseteq \mathfrak{m}_{\mathfrak{a}_{i}}$, for each $i \in\{1, \ldots, n\}$, and thus, by the Prime Avoidance Lemma, we can pick an element $x \in \mathfrak{m}$ such that $\left((x)+\mathfrak{a}_{i}\right)^{\star}=A$, for each $i=1, \ldots, n$. Now, set $\mathfrak{a}:=(x, a)^{\star}$. It is clear that $\mathfrak{a}$ is a proper $\star$-finite $\star$-ideal of $A$ (since 
$\mathfrak{a} \subseteq \mathfrak{m})$. For every $i=1, \ldots, n$, we have $A=\left((x)+\mathfrak{a}_{i}\right)^{\star} \subseteq\left(\mathfrak{a}+\mathfrak{a}_{i}\right)^{\star}$, and hence $\widehat{\mathscr{F}}:=\mathscr{F} \cup\{\mathfrak{a}\}$ is a $\star$-comaximal collection over $a$ of proper $\star$-finite $\star$-ideals of $A$. Moreover $\mathfrak{a} \neq \mathfrak{a}_{i}$, for each $i=1, \ldots, n$, since $x \in \mathfrak{a} \backslash \mathfrak{m}_{\mathfrak{a}_{i}}$ and $\mathfrak{a}_{i} \subseteq \mathfrak{m}_{\mathfrak{a}_{i}}$. Thus we have $\mathscr{F} \subsetneq \widehat{\mathscr{F}}$, a contradiction, by virtue of the maximality of $\mathscr{F}$ in $\left(\Sigma^{\prime}\right)_{a}^{\star}$.

The following remark allows us to get some sufficient condition about the existence of a collection of ideals as stated in Proposition 1.3.

Proposition 1.4. Let $A$ be an integral domain, $\star$ a star operation on $A$ and $a \in A \backslash\{0\}$. Assume that, for every ideal $\mathfrak{a} \in \mathcal{I}_{\text {fin }}^{\star}(A)$ containing $a$, there exists an ideal $\mathfrak{b} \in \mathcal{I}_{\text {fin }}^{\star}(A)$ with the property ( $\star$-c.a.) containing $\mathfrak{a}$. Then, the following statements hold.

(i) The family $\Sigma_{a}^{\star}$, partially ordered by the inclusion $\subseteq$, has maximal elements.

(ii) Every maximal element of $\Sigma_{a}^{\star}$ is a maximal element of $\left(\Sigma^{\prime}\right)_{a}^{\star}($ partially ordered by the same inclusion $\subseteq$ ).

(iii) There exists a maximal element of $\left(\left(\Sigma^{\prime}\right)_{a}^{\star}, \subseteq\right)$ which is an element of $\Sigma_{a}^{\star}$.

Proof. (i) By assumption, there exists a proper $\star$-finite $\star$-ideal $\mathfrak{b}$ with the property ( $\star$-c.a.) containing $a$. Then $\{\mathfrak{b}\} \in \Sigma_{a}^{\star}$. Now, let $\left\{\mathscr{G}_{\lambda}\right.$ : $\lambda \in \Lambda\} \subseteq \Sigma_{a}^{\star}$ be a chain and set $\mathscr{G}:=\bigcup_{\lambda \in \Lambda} \mathscr{G}_{\lambda}$. Clearly, every ideal in $\mathscr{G}$ has the property ( $\star$-c.a.). Now, let $\mathfrak{a}_{1}, \mathfrak{a}_{2} \in \mathscr{G}$. Then, pick elements $\lambda_{1}, \lambda_{2} \in \Lambda$ such that $\mathfrak{a}_{i} \in \mathscr{G}_{\lambda_{i}}(i=1,2)$. We can assume, without loss of generality, that $\mathscr{G}_{\lambda_{1}} \subseteq \mathscr{G}_{\lambda_{2}}$. Then, since $\mathscr{G}_{\lambda_{2}}$ is a $\star$-comaximal collection over $a$, we have $\left(\mathfrak{a}_{1}+\mathfrak{a}_{2}\right)^{\star}=A$. Hence $\mathscr{G} \in \Sigma_{a}^{\star}$ and thus it is an upper bound of the chain $\left\{\mathscr{G}_{\lambda}: \lambda \in \Lambda\right\}$. Now, statement (i) follows immediately by Zorn's Lemma.

(ii) Let $\mathscr{G}$ be a maximal element of $\Sigma_{a}^{\star}$. Assume, by contradiction, that there exists a collection $\mathscr{H} \in\left(\Sigma^{\prime}\right)_{a}^{\star}$ such that $\mathscr{G} \subsetneq \mathscr{H}$, and let $\mathfrak{a} \in \mathscr{H} \backslash \mathscr{G}$. Since $\mathscr{G} \cup\{\mathfrak{a}\} \notin \Sigma_{a}^{\star}$ is clearly a $\star$-comaximal collection over $a$ of proper $\star$-finite $\star$-ideals, it follows that the ideal $\mathfrak{a}$ has not the property ( $\star$-c.a.). By assumption, we can pick an ideal $\mathfrak{b} \in \mathcal{I}_{\text {fin }}^{\star}(A)$ containing $\mathfrak{a}$ such that $\mathfrak{b}$ satisfies the property ( $\star$-c.a.). Then $\mathfrak{b} \notin \mathscr{G}$, otherwise $\mathfrak{b} \in \mathscr{H}$, but $\mathfrak{b}$ is not $\star$-comaximal with $\mathfrak{a}$, and so this would be a contradiction. Thus, $\mathscr{G} \subsetneq \mathscr{\mathscr { G }}:=\mathscr{G} \cup\{\mathfrak{b}\}$. By construction, each ideal of $\widehat{\mathscr{G}}$ satisfies the property ( $\star$-c.a.). Moreover, every element of $\widehat{\mathscr{G}}$ is different by $\mathfrak{a}$, and hence, since $\mathscr{H}$ is a $\star$-comaximal collection over $a$, we have $A=(\mathfrak{c}+\mathfrak{a})^{\star} \subseteq(\mathfrak{c}+\mathfrak{b})^{\star}$. This shows that $\widehat{\mathscr{G}} \in \Sigma_{a}^{\star}$, a contradiction, by the maximality of $\mathscr{G}$. Thus (ii) is proved. Statements (iii) follows immediately by (i) and (ii). 
Proposition 1.5. Let $A$ be an integral domain, $\star$ a star operation on $A$ and $a \in A \backslash\{0\}$. Assume that every family $\mathscr{G} \in\left(\Sigma^{\prime}\right)_{a}^{\star}$ is finite. Then, for each $\mathfrak{a} \in \mathcal{I}_{\text {fin }}^{\star}(A)$, with $a \in \mathfrak{a}$, there exists an ideal $\mathfrak{b} \in \mathcal{I}_{\text {fin }}^{\star}(A)$ containing $\mathfrak{a}$ such that $\mathfrak{b}$ satisfies the condition ( $\star$-c.a.).

Proof. Assume, by a way of contradiction, that the statement is false. Then, in particular, $\mathfrak{a}$ does not satisfy the property ( $\star$-c.a.). Hence, there exist proper ideals $\mathfrak{a}_{1}^{1}, \mathfrak{a}_{2}^{1} \in \mathcal{I}_{\text {fin }}^{\star}(A)$ containing $\mathfrak{a}$ such that $\left(\mathfrak{a}_{1}^{1}+\right.$ $\left.\mathfrak{a}_{2}^{1}\right)^{\star}=A$. For each $i \geq 2$, by an easy induction argument, we can pick proper $\star$-finite $\star$-ideals $\mathfrak{a}_{1}^{i}, \mathfrak{a}_{2}^{i}$ containing $\mathfrak{a}_{1}^{i-1}$ such that $\left(\mathfrak{a}_{1}^{i}+\mathfrak{a}_{2}^{i}\right)^{\star}=A$. Now, fix $i \geq 2$ and $j<i$. Keeping in mind that

$$
\mathfrak{a}_{2}^{j} \supseteq \mathfrak{a}_{1}^{j-1} \supseteq \mathfrak{a}_{1}^{i},
$$

it follows immediately that $\left(\mathfrak{a}_{2}^{i}+\mathfrak{a}_{2}^{j}\right)^{\star}=A$. This proves that $\mathscr{F}:=\left\{\mathfrak{a}_{2}^{i}\right.$ : $i \geq 1\}$ is a $\star$-comaximal collection of $\star$-finite $\star$-ideals. Moreover, $\mathscr{F}$ is infinite, since each element of $\mathscr{F}$ is a proper $\star$-ideal. This gets a contradiction.

Proposition 1.6. Let $A$ be an integral domain and $\star$ a finite type star operation on $A$. Then the following conditions are equivalent.

(i) A has the $\star$-finite character.

(ii) For each nonzero element $a \in A$, every family $\mathscr{G} \in\left(\Sigma^{\prime}\right)_{a}^{\star}$ is finite.

Proof. (i) $\Rightarrow$ (ii) Fix a nonzero element $a \in A$ and let $\mathscr{F}$ be an element of $\left(\Sigma^{\prime}\right)_{a}^{\star}$. For every $\mathfrak{a} \in \mathscr{F}$, we can pick a $\star$-maximal $\star$-ideal $\mathfrak{m}(\mathfrak{a})$ containing $\mathfrak{a}$ (and $a$, in particular). Since $\mathscr{F}$ is a $\star$-comaximal collection, for distinct ideals $\mathfrak{a}, \mathfrak{b} \in \mathscr{F}$, we have that $\mathfrak{m}(\mathfrak{a}) \neq \mathfrak{m}(\mathfrak{b})$. Thus the cardinality of $\mathscr{F}$ is less than or equal to that of $\star-\operatorname{Max}(A) \cap V(a)$. Then (ii) follows by assumption.

(ii) $\Rightarrow$ (i) Fix a nonzero element $a \in A$. By assumption and Proposition 1.5, we can apply Proposition 1.4 (iii), and thus there exists a collection of ideals $\mathscr{F} \in \operatorname{Max}_{\subseteq}\left(\left(\Sigma^{\prime}\right)_{a}^{\star}\right) \cap \Sigma_{a}^{\star}$. Then the conclusion follows immediately by Proposition 1.3.

Let $A$ be an integral domain and $\mathcal{A}$ a family of overrings of $A$ such that $A=\bigcap \mathcal{A}$. For each $B \in \mathcal{A}$, let $\star_{B}$ be a star operation on $B$.

Recall by [1, Theorem 2], that

$$
I \mapsto I^{\wedge_{B \in \mathcal{A}^{\star} B}}:=\bigcap_{B \in \mathcal{A}}(I B)^{\star_{B}}
$$

is a star operation on $A$. Moreover, if each $\star_{B}$ is of finite type and the intersection $\bigcap \mathcal{A}$ is locally finite (i.e., each element $d \in \bigcap \mathcal{A}$ is a unit in all except a finite number of domains in $\mathcal{A}$ ), then $\wedge_{B \in \mathcal{A}} \star_{B}$ is of finite type. 
Proposition 1.7. Let $A$ be an integral domain and $\Delta \subseteq \operatorname{Spec}(A)$. Assume that $A=\bigcap_{\mathfrak{p} \in \Delta} A_{\mathfrak{p}}$ is a locally finite intersection. For each $\mathfrak{p} \in \Delta$, let $\star_{\mathfrak{p}}$ be a finite type star operation on $A_{\mathfrak{p}}$ and set $\star:=\wedge_{\mathfrak{p} \in \Delta \star_{\mathfrak{p}}}$. If $\mathfrak{a}$ is an ideal of $A$ such that $\mathfrak{a} A_{\mathfrak{p}}$ is $\star_{\mathfrak{p}}$-finite for each $\mathfrak{p}$, then $\mathfrak{a}$ is $\star$-finite.

Proof. As usual, take an $x \in \mathfrak{a}$. Let $\mathfrak{p}_{1}, \mathfrak{p}_{2}, \ldots, \mathfrak{p}_{n}$ the only primes in $\Delta$ containing $x$. So, if $\mathfrak{p} \neq \mathfrak{p}_{1}, \ldots, \mathfrak{p}_{n}, \mathfrak{a} A_{\mathfrak{p}}=x A_{\mathfrak{p}}=A_{\mathfrak{p}}$. Since the $\star_{\mathbf{p}}$ 's are of finite type, for each $i=1, \ldots, n$, we can find a finitely generated ideal $\mathfrak{a}_{i}$ of $A$, included in $\mathfrak{a}$, such that $\left(\mathfrak{a} A_{\mathfrak{p}}\right)^{\star_{\mathfrak{p}}}=\left(\mathfrak{a}_{i} A_{\mathfrak{p}}\right)^{\star_{\mathfrak{p}}}$. Let $\mathfrak{b}=(x)+\mathfrak{a}_{1}+\ldots+\mathfrak{a}_{n}$. Obviously $\mathfrak{b}$ is a finitely generated integral ideal of $A$ contained in $\mathfrak{a}$.

If $\mathfrak{p} \neq \mathfrak{p}_{1} \ldots \mathfrak{p}_{n}, \mathfrak{b} A_{\mathfrak{p}} \supseteq x A_{\mathfrak{p}}=A_{\mathfrak{p}}$ and so $\left(\mathfrak{b} A_{\mathfrak{p}}\right)^{\star_{\mathfrak{p}}}=\left(A_{\mathfrak{p}}\right)^{\star_{\mathfrak{p}}}=\left(\mathfrak{a} A_{\mathfrak{p}}\right)^{\star_{\mathfrak{p}}}$. Moreover, for $i=1,2, \ldots, n,\left(\mathfrak{a} A_{\mathfrak{p}_{i}}\right)^{\star_{\mathfrak{p}_{i}}}=\left(\mathfrak{a}_{i} A_{\mathfrak{p}_{i}}\right)^{\star_{p_{i}}} \subseteq\left(\mathfrak{b} A_{\mathfrak{p}_{i}}\right)^{\star_{p_{i}}} \subseteq$ $\left(\mathfrak{a} A_{\mathfrak{p}_{i}}\right)^{\star_{\mathfrak{p}_{i}}}$.

So, for each $\mathfrak{p} \in \Delta,\left(\mathfrak{a} A_{\mathfrak{p}}\right)^{\star_{\mathfrak{p}}}=\left(\mathfrak{b} A_{\mathfrak{p}}\right)^{\star_{\mathfrak{p}}}$. It follows that $\mathfrak{a}^{\star}=\mathfrak{b}^{\star}$ and $\mathfrak{a}$ is $\star$-finite.

We will denote by $t_{\mathfrak{p}}$ the $t$-operation of $A_{\mathfrak{p}}$. Recall that B.G. Kang proved in [11, Lemma 3.4(3)] that, for each ideal $\mathfrak{a},\left(\mathfrak{a} A_{\mathfrak{p}}\right)^{t_{\mathfrak{p}}}=\left(\mathfrak{a}^{t} A_{\mathfrak{p}}\right)^{t_{\mathfrak{p}}}$.

Proposition 1.8. Let $A$ be an integral domain, $\Delta \subseteq \operatorname{Spec}(A)$ such that $A=\bigcap_{\mathfrak{p} \in \Delta} A_{\mathfrak{p}}$ is a locally finite intersection. Then $t=\wedge_{\mathfrak{p} \in \Delta} t_{\mathfrak{p}}$.

Proof. Since the intersection $A=\bigcap_{\mathfrak{p} \in \Delta} A_{\mathfrak{p}}$ is locally finite and $t_{\mathfrak{p}}$ is of finite type for each $\mathfrak{p} \in \Delta$, by [1, Theorem 2] it follows that $\star:=\wedge_{\mathfrak{p} \in \Delta} t_{\mathfrak{p}}$ is a finite type star operation on $A$. Thus $\star \leq t$. Conversely, for an ideal $\mathfrak{a}$, we have that $\mathfrak{a}^{t} \subseteq \mathfrak{a}^{t} A_{\mathfrak{p}}$ for each $\mathfrak{p} \in \Delta$, so $\mathfrak{a}^{t} \subseteq \bigcap_{\mathfrak{p}} \mathfrak{a}^{t} A_{\mathfrak{p}} \subseteq$ $\bigcap_{\mathfrak{p}}\left(\mathfrak{a}^{t} A_{\mathfrak{p}}\right)^{t_{\mathfrak{p}}}=\bigcap_{\mathfrak{p}}\left(\mathfrak{a} A_{\mathfrak{p}}\right)^{t_{\mathfrak{p}}}=\mathfrak{a}^{\star}$ (the first equality follows by Kang's result). Thus $t \leq \star$. Hence $t=\star$.

Theorem 1.9. Let $A$ be an integral domain with the $t$-finite character. Then each $t$-locally $t$-finite $t$-ideal is $t$-finite.

Proof. It follows immediately from Proposition 1.7 and Proposition 1.8, taking $\Delta=t-\operatorname{Max}(A)$.

The following technical result will be crucial, in the following.

Lemma 1.10. Let $A$ be an integral domain, $\star$ be a finite type star operation on $A, \mathscr{F}$ a collection of ideals of $A$ such that $\bigcap \mathscr{F}$ contains a nonzero element $a \in A$. Set

$$
\mathfrak{a}:=\left\{x \in A: x \prod \mathscr{F}^{\prime} \subseteq a A \text {, for some finite subset } \mathscr{F}^{\prime} \subseteq \mathscr{F}\right\} .
$$

Then the following conditions hold: 
(a) let $F:=\left\{x_{1}, \ldots, x_{n}\right\}$ be a nonempty finite subset of $\mathfrak{a}$. For each $i=$ $1, \ldots, n$, let $\mathscr{F}_{x_{i}}$ be a finite subcollection of $\mathscr{F}$ such that $x_{i} \prod \mathscr{F}_{x_{i}} \subseteq$ $(a)$, and let $y \in\left(x_{1}, \ldots, x_{n}\right)^{\star}, \mathscr{F}^{\prime}:=\bigcup_{i=1}^{n} \mathscr{F}_{x_{i}}$. Then $y \prod \mathscr{F}^{\prime} \subseteq(a)$ and, in particular, we have

$$
\left(x_{1}, \ldots, x_{n}\right)^{\star} \subseteq \mathfrak{a} .
$$

(b) $\mathfrak{a}$ is a $a$-ideal.

(c) If $\mathscr{F}$ is a $\star$-comaximal collection over a of ideals of $A$, then for each $\mathfrak{m} \in \star-\operatorname{Max}(A)$, we have that:

$\mathfrak{a} A_{\mathfrak{m}}= \begin{cases}a A_{\mathfrak{m}} & \text { if } \mathfrak{m} \nsupseteq \mathfrak{b}, \text { for each } \mathfrak{b} \in \mathscr{F} \\ a\left(A: \mathfrak{b}_{0}\right) A_{\mathfrak{m}} & \text { if } \mathfrak{b}_{0} \text { is the unique ideal in } \mathscr{F} \text { contained in } \mathfrak{m}\end{cases}$

(d) If $\mathfrak{a}$ is $\star$-finite, then there exists a finite subcollection $\widehat{\mathscr{G}}$ of $\mathscr{F}$ such that

$$
\mathfrak{a}=\left\{x \in A: x \prod \widehat{\mathscr{G}} \subseteq a A\right\} .
$$

Proof. Firstly, we note that $\mathfrak{a}$ is an ideal of $A$, as it is immediately seen.

(a). As a matter of fact, we have

$y \prod \mathscr{F}^{\prime} \subseteq\left(\left(x_{1}, \ldots, x_{n}\right)^{\star} \prod \mathscr{F}^{\prime}\right)^{\star} \subseteq\left(\sum\left\{x_{i} \prod \mathscr{F}_{x_{i}}: i=1, \ldots, n\right\}\right)^{\star} \subseteq(a)$.

(b). Let $x \in \mathfrak{a}^{\star}$. Since $\star$ is a star operation of finite type, then there exist elements $f_{1}, \ldots, f_{n} \in \mathfrak{a}$ such that $x \in\left(f_{1}, \ldots, f_{n}\right)^{\star}$. Then by statement (a), $\left(f_{1}, \ldots, f_{n}\right)^{\star} \subseteq \mathfrak{a}$. Thus $\mathfrak{a}$ is a $\star$-ideal.

(c) Let $\mathfrak{m}$ be a $\star$-maximal ideal of $A$. Since $\mathscr{F}$ is a $\star$-comaximal collection, $\mathscr{F}_{\mathfrak{m}}:=\{\mathfrak{b} \in \mathscr{F}: \mathfrak{b} \subseteq \mathfrak{m}\}$ has cardinality at most one. For each $\mathfrak{b} \in \mathscr{F} \backslash \mathscr{F}_{\mathfrak{m}}$, we can pick an element $x_{\mathfrak{b}} \in \mathfrak{b} \backslash \mathfrak{m}$. If $\mathscr{F}_{\mathfrak{m}}$ is empty it is clear that $\mathfrak{a} A_{\mathfrak{m}}=a A_{\mathfrak{m}}$. In fact, let $\frac{x}{s} \in \mathfrak{a} A_{\mathfrak{m}}, x \in \mathfrak{a}$ and $s \in A \backslash \mathfrak{m}$. By definition, there exists a finite subcollection $\mathscr{F}^{\prime} \subseteq \mathscr{F}$ such that $x \prod \mathscr{F}^{\prime} \subseteq a A$. Then $\prod_{\mathfrak{b} \in \mathscr{F}^{\prime}} x_{\mathfrak{b}} \in A \backslash \mathfrak{m}$ and thus

$$
\frac{x}{s}=\frac{x \prod_{\mathfrak{b} \in \mathscr{F}^{\prime}} x_{\mathfrak{b}}}{s \prod_{\mathfrak{b} \in \mathscr{F} \prime} x_{\mathfrak{b}}}=\frac{a b}{s \prod_{\mathfrak{b} \in \mathscr{F} \prime} x_{\mathfrak{b}}} \in a A_{\mathfrak{m}}
$$

for some $b \in A$. Now, suppose that there is a unique ideal $\mathfrak{b}_{0} \in \mathscr{F}_{\mathfrak{m}}$. It is clear that $a\left(A: \mathfrak{b}_{0}\right) \subseteq \mathfrak{a}$, and hence $a\left(A: \mathfrak{b}_{0}\right) A_{\mathfrak{m}} \subseteq \mathfrak{a} A_{\mathfrak{m}}$. Conversely, let $\frac{x}{s} \in \mathfrak{a} A_{\mathfrak{m}} x \in \mathfrak{a}, s \in A \backslash \mathfrak{m}$ and let $\mathscr{F}^{\prime}$ be a finite subcollection of $\mathscr{\mathscr { F }}$ such that $x \prod \mathscr{F}^{\prime} \subseteq a A$. If $\mathfrak{b}_{0} \notin \mathscr{F}^{\prime}$, then the same argument given above shows that $\frac{\bar{x}}{s} \in a A_{\mathfrak{m}} \subseteq a\left(A: \mathfrak{b}_{0}\right) A_{\mathfrak{m}}$. If $\mathfrak{b}_{0} \in \mathscr{F}_{\mathfrak{m}}$, set $p:=\prod_{\mathfrak{b} \in \mathscr{F} \backslash\left\{\mathfrak{b}_{0}\right\}} x_{\mathfrak{b}}$, if $\mathscr{F}^{\prime} \backslash\left\{\mathfrak{b}_{0}\right\} \neq \emptyset, p:=1$ otherwise. Then $x p \mathfrak{b}_{0} \subseteq a A$ 
and $p \notin \mathfrak{m}$. It follows that $x p A \subseteq a\left(A: \mathfrak{b}_{0}\right)$ keeping in mind that $\left(A: \mathfrak{b}_{0}\right)$ is a always a fractional $\star$-ideal. Then we have

$$
\frac{x}{s}=\frac{x p}{s p} \in a\left(A: \mathfrak{b}_{0}\right) A_{\mathfrak{m}}
$$

and thus (c) is proved.

(d). Pick elements $x_{1}, \ldots, x_{r} \in \mathfrak{a}$ such that $\mathfrak{a}=\mathfrak{a}^{*}=\left(x_{1}, \ldots, x_{r}\right)^{\star}$. For each $i \in\{1, \ldots, r\}$, let $\mathscr{G}_{i}$ be a finite subcollection of $\mathscr{F}$ such that $x_{i} \prod \mathscr{G}_{i} \subseteq(a)$. Thus it is enough to choose $\widehat{\mathscr{G}}:=\bigcup_{i=1}^{r} \mathscr{G}_{i}$ and apply statement (a) to get the equality $\mathfrak{a}=\left\{x \in A: x \prod \mathscr{\mathscr { G }} \subseteq(a)\right\}$.

We recall that a domain $A$ is $v$-coherent if for any nonzero finitely generated ideal $I$ of $A, I^{-1}$ is $v$-finite that is, $I^{-1}=J^{v}$ for some $J \in$ $\mathbf{f}(A)$ ([5, Proposition 3.6]). Important classes of $v$-coherent domains are Noetherian domains, Mori domains, Prüfer domains, PvMD's, finite conductor domains (i.e., $(x) \cap(y)$ is finitely generated for each $x, y \in A$ ), coherent domains (i.e., the intersection of two finitely generated ideals is finitely generated). A domain $A$ is t-locally $v$-coherent if $A_{\mathfrak{m}}$ is $v$ coherent, for each $\mathfrak{m} \in t-\operatorname{Max}(A)$.

Theorem 1.11. Let $A$ be an integral domain which is t-locally $v$ coherent. Then the following conditions are equivalent.

(i) A has the t-finite character;

(ii) every family of $t$-finite, $t$-comaximal, $t$-ideals over a nonzero element $a \in A$ is finite;

(iii) every nonzero $t$-locally $t$-finite ideal $I$ (i.e., for any $\mathfrak{m} \in t$-Max $(A)$, $I_{\mathfrak{m}}$ is $t$-finite with respect to the $t$-operation of $\left.A_{\mathfrak{m}}\right)$ is $t$-finite.

Proof. The equivalence (i) $\Leftrightarrow$ (ii) is given by Proposition 1.6 and it holds more in general for any domain $A$ and by replacing $t$ with any finite type star operation.

(iii) $\Rightarrow$ (ii).

Let $\mathscr{F} \in \Sigma_{a}^{t}$ (where, we recall that $\Sigma_{a}^{t}$ is the set of collections of $t$-comaximal, $t$-finite $t$-ideals of $A$ containing $a$ ) and set

$$
\mathfrak{a}:=\left\{x \in A: x \prod \mathscr{F}^{\prime} \subseteq(a), \text { for some finite subset } \mathscr{F}^{\prime} \subseteq \mathscr{F}\right\} .
$$

By Lemma $1.10, \mathfrak{a}$ is a $t$-ideal of $A$ and, moreover, for each $t$-maximal ideal $\mathfrak{m}$ of $A$, we have

$\mathfrak{a} A_{\mathfrak{m}}= \begin{cases}a A_{\mathfrak{m}} & \text { if } \mathfrak{m} \nsupseteq \mathfrak{b}, \text { for each } \mathfrak{b} \in \mathscr{F} \\ a\left(A: \mathfrak{b}_{0}\right) A_{\mathfrak{m}} & \text { if } \mathfrak{b}_{0} \text { is the unique ideal in } \mathscr{F} \text { contained in } \mathfrak{m}\end{cases}$

Thus, if $\mathfrak{b}_{0}$ is the unique ideal of $\mathscr{F}$ contained in $\mathfrak{m}$, we have that $\mathfrak{a} A_{\mathfrak{m}}=a\left(A: \mathfrak{b}_{0}\right) A_{\mathfrak{m}}=\left(A_{\mathfrak{m}}: \mathfrak{b}_{0} A_{\mathfrak{m}}\right)$, and it is $t_{\mathfrak{m}}$-finite since $\mathfrak{b}_{0} A_{\mathfrak{m}}$ is $t_{\mathfrak{m}}$ finite (see [11, Lemma 3.4(3)]) and $A_{\mathfrak{m}}$ is $v$-coherent. This shows that 
$\mathfrak{a}$ is a $t$-locally $t$-finite, $t$-ideal and hence it is $t$-finite by assumption. Again by Lemma 1.10, it follows that

$$
\mathfrak{a}=\left\{x \in A: x \prod \widehat{\mathscr{G}} \subseteq(a)\right\},
$$

for some finite subcollection $\widehat{\mathscr{G}}$ of $\mathscr{F}$. Now, consider an ideal $\mathfrak{b}_{1} \in \mathscr{F} \backslash \widehat{\mathscr{G}}$. By the definition of $\mathfrak{a}$, it follows immediately that $a\left(A: \mathfrak{b}_{1}\right) \subseteq \mathfrak{a}$, and thus $a\left(A: \mathfrak{b}_{1}\right) \prod \widehat{\mathscr{G}} \subseteq a A$, that is, $\left(A: \mathfrak{b}_{1}\right) \prod \widehat{\mathscr{G}} \subseteq A$. Hence we have that $\prod \widehat{\mathscr{G}} \subseteq\left(A:\left(A: \mathfrak{b}_{1}\right)\right)=\mathfrak{b}_{1}^{v}=\mathfrak{b}_{1}$, keeping in mind that $\mathfrak{b}_{1}$ is a $t$-finite $t$-ideal, so it is divisorial. Since $\mathscr{F}$ is a $t$-comaximal collection of ideals and $\mathfrak{b}_{1} \in \mathscr{F} \backslash \widehat{\mathscr{G}}$, we have that

$$
A=A^{t}=\left(\prod_{\mathfrak{b} \in \widehat{\mathscr{G}}}\left(\mathfrak{b}_{1}+\mathfrak{b}\right)^{t}\right)^{t}=\left(\prod_{\mathfrak{b} \in \widehat{\mathscr{G}}}\left(\mathfrak{b}_{1}+\mathfrak{b}\right)\right)^{t}=\left(\prod^{\hat{\mathscr{G}}}+\mathfrak{c}\right)^{t}
$$

for some ideal $\mathfrak{c} \subseteq \mathfrak{b}_{1}$. Finally, using the fact that $\prod \widehat{\mathscr{G}} \subseteq \mathfrak{b}_{1}$, it follows that

$$
A \subseteq\left(\prod \widehat{\mathscr{G}}+\mathfrak{c}\right)^{t} \subseteq \mathfrak{b}_{1}^{t}=\mathfrak{b}_{1}
$$

This proves that $\{A\}=\mathscr{F} \backslash \widehat{\mathscr{G}}$. Since $\widehat{\mathscr{G}}$ is finite, the statement follows.

(i) $\Rightarrow$ (iii) See Theorem 1.9, and observe that it holds for any domain (without the $t$-locally $v$-coherence hypothesis) .

Remarks 1.12. (a) A t-locally $v$-coherent domain is not necessarily a $\mathrm{P} v \mathrm{MD}$. In fact any Noetherian domain is t-locally $v$-coherent (and it is not always $\mathrm{P} v \mathrm{MD}$ ). This shows that Theorem 1.11 strictly generalizes the results obtained for Prüfer domains and PvMD's ([9, Proposition 6.11] and [15, Proposition 5]).

(b) More generally a $t$-locally $v$-coherent domain is not necessarily $v$ coherent (while a $v$-coherent domain is locally $v$-coherent). The example of a locally $v$-coherent domain that is not $v$-coherent given in [6, Example 3.3] is easily seen to be also $t$-locally $v$-coherent. However, as a consequence of Proposition 1.9, a $t$-locally $v$-coherent domain with $t$-finite character is $v$-coherent (cf. with [6, Proposition 3.1], where it is mentioned that locally $v$-coherent domains with finite character are $v$-coherent). So, the domains of Theorem 1.11 are in fact $v$-coherent.

(c) Note that by Proposition 1.7 and Proposition 1.8 it follows easily that if $A$ can be written as a locally finite intersection of $v$-coherent localizations, then $A$ is $v$-coherent. This generalizes both the results in (b) and [6, Proposition 3.1]. 
(d) The main hypothesis of Theorem 1.11 stating that $A$ is $t$-locally $v$-coherent is not always required in order to get the equivalences (i)-(ii)-(iii). In fact, take $A$ to be a pseudo-valuation domain with maximal ideal $M$ and such that $M$ is not principal as an ideal of $M^{-1}=V$. This is equivalent to requiring that $A$ is not $v$-coherent ([6, Remark 3.14]). It is well-known that $M$ is a $t$-ideal of $A$, so $A$ is not a $t$-locally $v$-coherent domain, but conditions (i)-(ii)-(iii) are trivially satisfied.

(e) In general, Theorem 1.11 cannot be extended to any finite type star operation. For instance, if we take the identity operation $d$ at the place of $t$, Theorem 1.11 fails. In fact, a Noetherian domain do not need to have the finite character on maximal ideals, but each locally finitely generated ideal is finitely generated.

We have already observed that Noetherian domains may not have the finite character, but they always have the $t$-finite character (since, more generally, Mori domains have the $t$-finite character [3, Proposition $2.2]$ ). Conversely, the following example shows that there exist domains with the finite character which do not have the $t$-finite character.

Example 1.13. We consider the following pullback diagram:

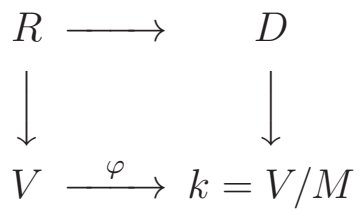

where $V$ is a valuation domain with maximal ideal $M$, residue field $k$ and $D$ is a 2-dimensional, local Krull domain with quotient field $k$.

The domain $R$ turns out to be local. By [6, Theorem 2.18] the $t$ maximal ideals of $R$ are the inverse images of the $t$-maximal ideals of $D$ (which are the height-one primes) and they all contain $M$. Thus $R$ does not have the $t$-finite character. But $R$ has the finite character as being local.

\section{THE LOCAL CHARACTER OF $\star$-INVERTIBILITY}

The following result has been proved by M. Zafrullah [15, Proposition $4]$ for the $t$-operation. We give a different proof of Zafrullah's result and generalize it to every star operation of finite type. The proof of the following Proposition uses an argument similar to the one given in the proof of Theorem 1.11.

Proposition 2.1. Let $A$ be an integral domain, $\star$ be a star operation of finite type on $A$ and $a \in A \backslash\{0\}$. Assume that every $\star$-locally 
principal ideal of $A$ is $\star$-invertible. Then every $\star$-comaximal collection over a of $\star$-invertible $\star$-ideals is finite.

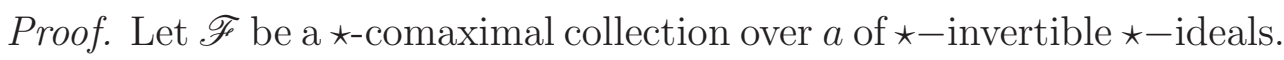
Now, let

$$
\mathfrak{a}:=\left\{x \in A: x \prod \mathscr{F}^{\prime} \subseteq(a), \text { for some finite subset } \mathscr{F}^{\prime} \subseteq \mathscr{F}\right\} .
$$

By Lemma 1.10 and the fact that every ideal in $\mathscr{F}$ is $\star$-invertible, it follows that $\mathfrak{a}$ is a $\star$-locally principal $\star$-ideal, and hence it is $\star$-finite, since it is $\star$-invertible, by assumption. Thus, by Lemma $1.10(\mathrm{c})$, there exists a finite subcollection $\widehat{\mathscr{G}}$ of $\mathscr{F}$ such that

$$
\mathfrak{a}=\left\{x \in A: x \prod \widehat{\mathscr{G}} \subseteq(a)\right\} .
$$

Now, consider an ideal $\mathfrak{b}_{1} \in \mathscr{F} \backslash \widehat{\mathscr{G}}$. By the definition of $\mathfrak{a}$, it follows immediately that $a\left(A: \mathfrak{b}_{1}\right) \subseteq \mathfrak{a}$, and thus $a\left(A: \mathfrak{b}_{1}\right) \prod \widehat{\mathscr{G}} \subseteq a A$, that is, $\left(A: \mathfrak{b}_{1}\right) \prod \widehat{\mathscr{G}} \subseteq A$. Hence we have

$$
\prod \widehat{\mathscr{G}}=\prod \widehat{\mathscr{G}}\left(\left(A: \mathfrak{b}_{1}\right) \mathfrak{b}_{1}\right)^{\star} \subseteq\left(\prod \widehat{\mathscr{G}}\left(A: \mathfrak{b}_{1}\right) \mathfrak{b}_{1}\right)^{\star} \subseteq \mathfrak{b}_{1}^{\star}=\mathfrak{b}_{1},
$$

keeping in mind that $\mathfrak{b}_{1}$ is a $\star$-invertibile $\star$-ideal. Since $\mathscr{F}$ is a $\star$ comaximal collection of ideals and $\mathfrak{b}_{1} \in \mathscr{F} \backslash \widehat{\mathscr{G}}$, we have

$$
A=A^{\star}=\left(\prod_{\mathfrak{b} \in \widehat{\mathscr{G}}}\left(\mathfrak{b}_{1}+\mathfrak{b}\right)^{\star}\right)^{\star}=\left(\prod_{\mathfrak{b} \in \widehat{\mathscr{G}}}\left(\mathfrak{b}_{1}+\mathfrak{b}\right)\right)^{\star}=\left(\prod \widehat{\mathscr{G}}+\mathfrak{c}\right)^{\star}
$$

for some ideal $\mathfrak{c} \subseteq \mathfrak{b}_{1}$. Finally, using the fact that $\prod \widehat{\mathscr{G}} \subseteq \mathfrak{b}_{1}$, we have

$$
A \subseteq\left(\prod \widehat{\mathscr{G}}+\mathfrak{c}\right)^{\star} \subseteq \mathfrak{b}_{1}^{\star}=\mathfrak{b}_{1}
$$

This proves that $\{A\}=\mathscr{F} \backslash \widehat{\mathscr{G}}$. Since $\widehat{\mathscr{G}}$ is finite, the statement follows.

Proposition 2.2. Let $A$ be a domain in which each t-locally principal t-ideal is t-finite. Then, for any star operation of finite type, each $\star$-locally principal $\star-i d e a l$ is $\star$-finite. In particular, a locally principal ideal is finitely generated (and so, invertible).

Proof. Let $I$ be a $\star$-ideal of $A$ that is $\star$-locally principal. Then $I$ is also $t$-locally principal since a $t$-ideal is in particular a $\star$-ideal and each $t$-maximal ideal is contained in a $\star$-maximal ideal. So $I$ is $t$ finite, say $I:=\left(a_{1}, \cdots, a_{n}\right)^{t}$, for some $a_{1}, \cdots, a_{n} \in I$. We show that $I$ is $\star$-invertible, that is $\left(I I^{-1}\right)^{\star}=A$, and so it is $\star$-finite. To see this, it 
is enough to show that for each $\star-$ maximal ideal $\mathfrak{m}$ of $A,\left(I I^{-1}\right)_{\mathfrak{m}}=A_{\mathfrak{m}}$. Now, if $I A_{\mathfrak{m}}=a A_{\mathfrak{m}}$, we have that:

$$
\begin{aligned}
A_{\mathfrak{m}} \supseteq\left(I I^{-1}\right)_{\mathfrak{m}} & =I_{\mathfrak{m}} I_{\mathfrak{m}}^{-1}=a A_{\mathfrak{m}}\left(a_{1}^{-1} A \cap \cdots \cap a_{n}^{-1} A\right)_{\mathfrak{m}} \\
& =a a_{1}^{-1} A_{\mathfrak{m}} \cap \cdots \cap a a_{n}^{-1} A_{\mathfrak{m}} \supseteq A_{\mathfrak{m}} .
\end{aligned}
$$

This proves the statement.

Proposition 2.2 does not hold if we replace the $t$-operation with another finite type star operation $\star$. This is what we show, for instance, in the next example with $\star=d$.

Example 2.3. Consider the domain $R$ constructed in Example 1.13. Since $R$ is local, each locally principal ideal is invertible.

By [6, Theorem 4.13] $R$ is a PvMD, so it is t-locally $v$-coherent. Since $R$ does not have the $t$-finite character, then there exists a nonzero ideal $I$ that is $t$-locally principal but not $t$-finite.

Now we look more closely to the proof of Proposition 2.1 and show that this result can be slightly improved in the case of the $t$-operation. We will call an ideal $\mathfrak{a}$ t-principal if $\mathfrak{a}^{t}$ is principal.

The fact that the family $\mathscr{F}$ is composed of $\star$-invertible ideals is used twice in the proof. First, to show that $a A_{\mathfrak{m}}=a\left(A: \mathfrak{b}_{0}\right) A_{\mathfrak{m}}$ is a principal ideal. Here, it is clear that it is sufficient to assume that $\left(A: \mathfrak{b}_{0}\right)$ is principal when localized at the $\star$-maximal ideals. If we assume $\mathfrak{b}_{0}$ to be $t$-finite, it is easily seen that $\left(A: \mathfrak{b}_{0}\right) A_{\mathfrak{m}}=\left(A_{\mathfrak{m}}: \mathfrak{b}_{0} A_{\mathfrak{m}}\right)$. So $\left(A: \mathfrak{b}_{0}\right) A_{\mathfrak{m}}$ is principal if and only if $\left(\mathfrak{b}_{0} A_{\mathfrak{m}}\right)^{t}=\left(\mathfrak{b}_{0} A_{\mathfrak{m}}\right)^{v}$ is principal. Thus, in this point of the proof, we use only the fact that $\mathfrak{b}_{0}$ is $t$-finite and $\mathfrak{b}_{0} A_{\mathfrak{m}}$ is $t$-principal.

The other point of the proof where $\star$-invertibility for ideals of $\mathscr{F}$ is used is where from the containment $\left(A: \mathfrak{b}_{1}\right) \prod \widehat{\mathscr{G}} \subseteq A$ we deduce that $\prod \widehat{\mathscr{G}} \subseteq \mathfrak{b}_{1}$. But this is true in our case since $\mathfrak{b}_{1}$ is a $t$-finite $t$-ideal.

In conclusion, for the $t$-operation, Proposition 2.1 can be modified as follows:

Proposition 2.4. Let $A$ be an integral domain and $a \in A \backslash\{0\}$. Assume that every $t$-locally principal ideal of $A$ is $t$-invertible. Then every $t$-comaximal collection over a of $t$-finite $t$-locally $t$-principal $t$ ideals is finite.

We give an application of this result, to obtain a further generalization of Bazzoni's conjecture.

Recall that if $\left(x_{1}, \ldots, x_{n}\right)_{v}=(x)$ for some $x$ in $A$, then $x$ is called the $v$-ged of $x_{1}, \ldots, x_{n}$ and all pairs of elements of $A$ have a $v$-ged if and only if $D$ is a GCD-domain. 
Proposition 2.5. For a domain $A$ the following conditions hold:

(1) if $A$ is a GCD domain then it is v-coherent.

(2) if $A$ is a t-locally GCD domain, then a nonzero $t$-finite $t$-ideal of $A$ is t-locally t-principal.

Proof. (1) It follows from the fact that a GCD domain is a $\mathrm{P} v \mathrm{MD}$, and $\mathrm{P} v \mathrm{MD}$ 's are $v$-coherent

(2) Let $\mathfrak{a}$ be a $t$-finite $t$-ideal and $\mathfrak{m}$ a $t$-maximal ideal. Let $\mathfrak{b}$ be a finitely generated ideal of $D$ such that $\mathfrak{b}^{t}=\mathfrak{a}$ and let $x$ be a $v$-GCD of the generators of $\mathfrak{b} A_{\mathfrak{m}}$. We have

$$
\left(\mathfrak{a} A_{\mathfrak{m}}\right)^{t_{\mathfrak{m}}}=\left(\mathfrak{b}^{t} A_{\mathfrak{m}}\right)^{t_{\mathfrak{m}}}=\left(\mathfrak{b} A_{\mathfrak{m}}\right)^{t_{\mathfrak{m}}}=\left(x A_{\mathfrak{m}}\right)^{t_{\mathfrak{m}}} .
$$

Thus, $\mathfrak{a}$ is $t$-locally $t$-principal.

Proposition 2.6. Let $A$ be a t-locally GCD domain. If every t-locally principal ideal of $D$ is t-invertible then $D$ has the t-finite character.

Proof. Note that by Proposition 2.5(2), $A$ is t-locally $v$-coherent. Let $a$ be an element of $A$. Take a $t$-comaximal collection $\mathcal{F}$ over $a$ of proper $t$-finite $t$-ideals. By Proposition $2.5, \mathcal{F}$ is in particular a $t$-comaximal collection over $a$ of $t$-finite $t$-locally $t$-principal $t$-ideals. So, it is finite by Proposition 2.4. Hence $D$ has the $t$-finite character by Theorem 1.11 .

Proposition 2.6 extends the $t$-version of Bazzoni's conjecture ([9, Proposition 6.11] and [15, Proposition 5]) to t-locally GCD domains. Note that locally GCD domains are not necessarily P $v$ MD ([14, Example 2.6]). Now, if $\mathfrak{p}$ is a $t$-maximal ideal of a locally GCD domain $A$, there exists a maximal ideal $\mathfrak{m}$ of $A$ such that $\mathfrak{p} \subseteq \mathfrak{m}$. So $A_{\mathfrak{p}}$ is a localization of the GCD domain $A_{\mathfrak{m}}$, and so it is a GCD domain. Thus a locally GCD domain is also $t$-locally GCD and so the class of $t$-locally GCDs is properly larger than the class of PvMDs.

However, we can prove that a $t$-locally GCD domain $A$ such that every $t$-locally principal ideal of $A$ is $t$-invertible is a $\mathrm{P} v \mathrm{MD}$. More precisely, we will show that a $t$-locally GCD domain with the $t$-finite character is a $\mathrm{P} v \mathrm{MD}$.

First we notice that, slightly modifying the proof of Proposition 2.1, similarly to as we have done to obtain Proposition 2.4, we have the following result.

Proposition 2.7. Let $A$ be an integral domain and $a \in A \backslash\{0\}$. Assume that every t-locally t-invertible t-ideal of $A$ is t-invertible. Then every t-comaximal collection of $t$-locally $t$-invertible $t$-ideals over $a$ is finite. 
Proposition 2.8. Let $A$ be a t-locally PvMD. The following are equivalent:

(i) Each t-locally t-invertible t-ideal is t-invertible.

(ii) A has the t-finite character.

(iii) A is a PvMD with the t-finite character.

Proof. (iii) $\Rightarrow$ (ii) It is trivial.

(ii) $\Rightarrow$ (i) This follows from Theorem 1.9 for any integral domain $A$.

(i) $\Rightarrow$ (iii) Let $I$ be a $t$-finite $t$-ideal of $A$. Then $I$ is $t$-locally finite, so it is $t$-locally $t$-invertible (since $A$ is a $t$-locally $\mathrm{P} v \mathrm{MD}$ ) and, by assumption (i), $I$ is $t$-invertible. This shows that $A$ is a $\mathrm{P} v \mathrm{MD}$.

Now, let $\mathscr{F}$ be a $t$-comaximal collection of $t$-finite $t$-ideals (over a nonzero element $a \in A$ ). Then, by the fact that $A$ is a $t$-locally $\mathrm{P} v \mathrm{MD}$, $\mathscr{F}$ is also a $t$-comaximal family of $t$-locally $t$-invertible $t$-ideals. By assumption (i) and by Proposition 2.7, it follows that $\mathscr{F}$ is finite. Since a $\mathrm{P} v \mathrm{MD}$ is $v$-coherent, $A$ has the $t$-finite character by Theorem 1.11.

We easily have the following corollary.

Corollary 2.9. Let $A$ be a t-locally GCD domain. The following are equivalent:

(i) Each t-locally principal t-ideal is t-invertible.

(ii) A has the t-finite character.

(iii) A is a PvMD with the t-finite character.

\section{REFERENCES}

[1] D.D. Anderson, Star-operations induced by overrings, Comm. Algebra 16 (1988), no. 12, 2535-2553.

[2] D.D. Anderson And D.F. Anderson, Some remarks on cancellation ideals. Math. Japon, 29 no. 6 (1984), 879-886.

[3] V. Barucci And S. GABelli, How far is a Mori domain from being a Krull domain?, J. Pure Appl. Algebra 45 (1987), no. 2, 101-112.

[4] S. BAzzoni, Class semigroups of Prüfer domains, J. Algebra 184 (1996), 613631.

[5] M. Fontana And S. Gabelli, On the class group and the local class group of a pullback, J. Algebra 181 (1996), no. 3, 803-835

[6] S. Gabelli and E.G. Houston, Coherent-like conditions in pullbacks, Michigan Math. J. 44 (1997), no. 1, 99-123.

[7] S. Glaz And W.V. Vasconcelos, Flat ideals. II, Manuscripta Math. 22 no.4 (1977), 325-341.

[8] S. Glaz And W.V. Vasconcelos, Flat ideals. III, Comm. Algebra 12 no.2 (1984), 199-227. 
[9] F. Halter-Koch, Clifford semigroups of ideals in monoids and domains, Forum Math., to appear.

[10] W.C. Holland, J. Martinez, W.Wm. McGovern and M.Tesemma, Bazzoni's Conjecture, J. Algebra 320 (2008), 1764-1768.

[11] B. G. KAng, Prüfer v-multiplication domains and the ring $R[X]_{N_{v}}$, J. Algebra 123 (1989), no. 1, 151-170.

[12] W. Wm. McGovern, Prufer domains with Clifford Class semigroup, J. Comm. Alg., to appear.

[13] G.Picozza and F.Tartarone, Flat ideals and stability in integral domains, preprint.

[14] M. Zafrullah, The $D+X D_{S}[X]$ construction from $G C D$-domains, J. Pure Appl. Algebra 50 (1988), no. 1, 93-107.

[15] Muhammad Zafrullah, t-Invertibility and Bazzoni-like statements, J. Pure Appl. Algebra, to appear.

Dipartimento di Matematica, Università degli studi Roma Tre, Largo San Leonardo Murialdo 1, 00146 Roma, Italy

E-mail address: carmelo@mat.uniroma3.it

Laboratoire dAnalyse, Topologie, Probabilits, Faculté des Sciences de Marseille Saint-Jerôme 13397 Marseille Cedex 20, France

E-mail address: giampaolo.picozza@univ-cezanne.fr

Dipartimento di Matematica, Università degli studi Roma Tre, Largo San LeOnardo Murialdo 1, 00146 Roma, Italy

E-mail address: tfrance@mat.uniroma3.it 